\title{
A Program Evaluation of the Engagement of Participants in the AstraZeneca COVID-19 Vaccine Clinical Trial at Indiana University School of Medicine Cynthia Ziwawo', Sylk Sotto-Santiago ${ }^{2}$ \\ ${ }^{1}$ Indiana University School of Medicine; ${ }^{2}$ Indiana University School of Medicine, Department of Medicine
}

\section{Background/Objective:}

Since the emergence of various COVID-19 vaccines, there have been a significant amount of members of historically marginalized populations that remain unvaccinated. Specifically, Black and Latino/x populations vaccine rates are consistently lower than their white counterparts not just in Indiana, but across the country. The recruitment of the AstraZeneca clinical trial for the COVID-19 vaccine that was hosted by Indiana University School of Medicine underscored this disparity. The drop off rates for Black and Latino/x participants between being screened for the study to ultimately being enrolled into the study became a cause for concern. The concept of vaccine hesitancy in these communities is a concept worth further exploration in order to determine the best strategies to improve health outcomes as we continue to mitigate the current pandemic.

\section{Methods:}

Interviews with the research assistants of the AstraZeneca clinical trial were conducted in order to identify the major causes of hesitancy and acceptance amongst the individuals that were screened and enrolled into the study. The questions explored the interactions with the participants, specifically for anecdotal evidence of the screened participants that ultimately did and did not participate in the study.

\section{Results:}

Despite the outpouring of Indiana residents that enrolled in the study, many chose not to participate due to unknown potential side effects of the vaccine, access to provider advise, trust, among others. Furthermore, the timeframe of the study was heavily impacted by the availability of the FDA approval of Pfizer's vaccine, in which many participants chose to drop out for a guaranteed vaccine.

\section{Conclusion and Potential Impact:}

Identifying barriers to vaccine acceptance in marginalized communities will provide vital information for advancing public health efforts to increase vaccination rates. Additionally, implementing these strategies into vaccine clinical trials will allow for more equitable representation, and culturally competent scientific evidence. 